\title{
Cortical pore size distribution and viscoelastic tibia properties discriminate fragility fractures independent of bone mineral density
}

Kay Raum ( $\sim$ kay.raum@charite.de )

Charité-Universitätsmedizin Berlin https://orcid.org/0000-0003-0573-1622

Gabriele Armbrecht

Charité-Universitätsmedizin Berlin

Huong Minh

Charité-Universitätsmedizin Berlin

Jonas Massmann

Charité-Universitätsmedizin Berlin

\section{Article}

Keywords: osteoporosis, bone disorders, bone mass, bone fragility

Posted Date: May 12th, 2021

DOI: https://doi.org/10.21203/rs.3.rs-515087/v1

License: (c) (i) This work is licensed under a Creative Commons Attribution 4.0 International License. Read Full License 


\title{
Cortical pore size distribution and viscoelastic tibia properties discriminate fragility fractures independent of bone mineral density
}

\author{
Gabriele Armbrecht ${ }^{1}$, Huong Nguyen Minh ${ }^{2}$, Jonas Massmann ${ }^{2}$, Kay Raum ${ }^{2 *}$ \\ ${ }^{1}$ Charité-Universitätsmedizin Berlin, Corporate Member of Freie Universität \\ Berlin, Humboldt-Universität zu Berlin, and Berlin Institute of Health, Center for \\ Muscle and Bone Research, 12203 Berlin, Germany \\ ${ }^{2}$ Charité-Universitätsmedizin Berlin, Corporate Member of Freie Universität \\ Berlin, Humboldt-Universität zu Berlin, and Berlin Institute of Health, Berlin \\ Institute of Health Center for Regenerative Therapies, 13353 Berlin, Germany
}

\begin{abstract}
Osteoporosis is a disorder of bone remodeling leading to reduced bone mass, structural deterioration, and increased bone fragility. The established diagnosis is based on the measurement of areal bone mineral density by dual energy $x$-ray absorptiometry (DXA), which poorly captures individual bone loss and structural decay. Enlarged cortical pores in the tibia have been proposed to indicate structural deterioration and reduced bone strength in the hip. Here, we report for the first time the in-vivo assessment of the cortical pore size distribution together with viscoelastic bone properties by means of a novel ultrasonic cortical backscatter (CortBS) technology to discriminate between fractured and nonfractured postmenopausal women $(\mathrm{N}=55)$. The discrimination performance was benchmarked with DXA and high-resolution peripheral computed tomography (HR-pQCT). The results suggest a superior discrimination performance of CortBS (area under the receiver operating characteristic curve: $0.69 \leq \mathrm{AUC} \leq 0.75)$ compared to DXA $(0.53 \leq \mathrm{AUC} \leq 0.55)$ and a similar performance compared to HRPQCT $(0.68 \leq \mathrm{AUC} \leq 0.73)$.
\end{abstract}




\section{Introduction}

Osteoporosis (OP) is an age-associated disorder of bone remodeling leading to reduced bone mass, structural deterioration, and increased bone fragility ${ }^{1}$. According to a recent systematic review of burden and management of fragility fractures in the largest EU countries ${ }^{2}$, fragility fractures caused by OP are the $4^{\text {th }}$ leading cause of chronic disease morbidity. Although OP is generally thought of as a "woman's disease" ${ }^{3}$, men account for a third of OP-related hip fractures in Europe ${ }^{4}$. An estimated 1.0 million quality-adjusted life years (QALYS) were lost in 2017 due to fragility fractures. The current disability-adjusted life years (DALYS) of individuals age $>50$ years were higher than those for stroke ${ }^{2}$. Established guidelines for the diagnosis of OP recommend the assessment of fracture risk factors and the T-score, which is derived from the measurement of areal bone mineral density ( $a B M D)$ by means of dual energy $x$-ray absorptiometry (DXA) at major fracture sites, i.e., spine and proximal femur ${ }^{5}$. OP is defined for $a B M D$ values $2.5 \mathrm{SD}$ or more below the mean assessed in young adults (i.e., T-score $\leq$ 2.5). Low bone mass (osteopenia, $-1 \geq T$-score $>-2.5$ ) is currently not considered as a disease ${ }^{5}$, although a specific osteoprotective therapy is recommended if additional clinical risk factors are present ${ }^{6}$. However, bone strength is determined by a plethora of factors including size, shape, architecture, and composition ${ }^{7}$. Today, there is increasing evidence that the majority of individuals who have sustained an osteoporosis-related fracture or who are at high risk of fracture are not diagnosed as osteoporotic according to the $B M D$ level ${ }^{8,9}$ (Fig. 1).

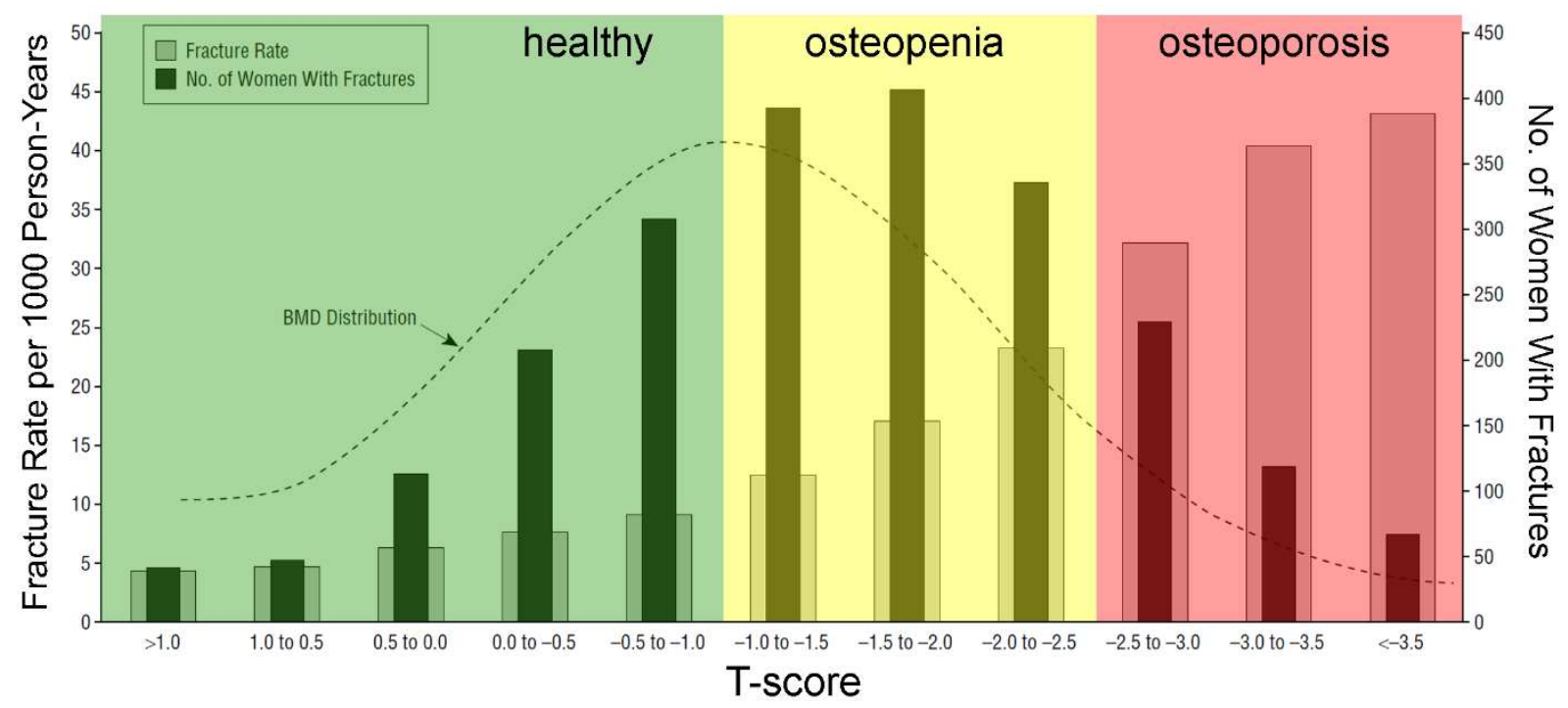

Fig. 1 Distribution of fracture rate and absolute number of women with fractures vs. bone mineral density (expressed as $T$ score values) from the National Osteoporosis Risk Assessment (NORA) study ${ }^{9}$. In this longitudinal observational study $N=149.524$ postmenopausal women (age range: $50-104 \mathrm{yrs}$ ) were evaluated. The colors indicate the established diagnosis thresholds. $82 \%$ of women with fractures had a T-score higher than -2.5, i.e., they are not considered to have osteoporosis according to the BMD level. Adapted from ${ }^{10}$ under a Creative Commons Attribution 2.0 License.

Bone tissue undergoes permanent remodeling. Under normal conditions, osteoclasts create resorption canals in the cortical bone tissue matrix, which are refilled by osteoblasts leaving a Haversian canal with a diameter of approximately $30 \mu \mathrm{m}{ }^{11}$. Bone loss occurs in both women and men as part of the natural aging process ${ }^{12}$. Unbalanced intracortical remodeling typically starts in the endosteal subcompartment and leaves partially refilled or even non-refilled bone multicellular units (BMUs). Clustering of BMUs enhances their chances to merge, creating "giant" pores with diameters larger than 
$385 \mu \mathrm{m}^{13}$, leading to the so-called trabecularized cortex ${ }^{14}$, and ultimately to a thinning of the cortical bone shell. However, more than $60 \%$ of intracortical pores are smaller than $100 \mu \mathrm{m}$ in diameter ${ }^{14}$. In the femoral neck of elderly people (age between 60 and 90 years), decreases of cortical thickness (Ct.Th) and porosity (Ct.Po) by $\sim 4 \%$ and $\sim 32 \%$, respectively, per decade with no significant changes in pore density (Ct.Po.Dn) have been reported ${ }^{15}$. Decreased cortical thickness and the prevalence of large BMU's have been shown to reduce bone strength ${ }^{16}$ and structural deterioration ${ }^{17}$. In contrast, cortical thinning is partially compensated by the apposition of new tissue matrix at the periosteal interface leading to an increased bone diameter and a further increase of the pore diameter gradient in the radial bone direction. Therefore, the cortical pore diameter distribution Ct.Po.Dm.D (hereinafter simply called pore size distribution) represents a relevant target for identifying patients with high fracture risk. However, given their small size, the in-vivo imaging of cortical pores remains challenging, even with the most advanced high-resolution peripheral quantitative computed tomography (HRpQCT). The imaging resolution of the first and second-generation HR-pQCT systems with voxel sizes of 82 and $61 \mu \mathrm{m}$ allows direct visualization and segmentation of large pores (i.e., Ct.Po.Dm >100 $\mu \mathrm{m}$ ) only, leaving the major fraction of smaller cortical pores unresolved. lori et al. ${ }^{18}$ have proposed a calibration rule for the estimation of Ct.Po locally from volumetric BMD (VBMD) distribution parameters. This method is more accurate (absolute error: $3.4 \%$ ) than established $V B M D$ or thresholdbased approaches, as it approximates the contribution of unresolved pores (i.e., Ct.Po.Dm $<80 \mu \mathrm{m}$ ). However, the HR-pQCT technology is not widely distributed and is used mostly in clinical research so far. With DXA-based $a B M D$, i.e., the diagnostic gold-standard, cortical bone loss resulting from structural decay are poorly captured ${ }^{9,19,20}$.

Quantitative ultrasound (QUS) methods are non-ionizing alternatives for the diagnosis of osteoporosis and the prediction of fracture risk ${ }^{21}$. Many QUS approaches target trabecular sites, e.g. at the heel, and predicting $B M D$ via empirical associations with the measured speed of sound (SOS), broadband ultrasound attenuation (BUA) and other parameters derived from the measurement ${ }^{22}$. More recent QUS technologies, e.g., bidirectional axial transmission measure cortical sites, e.g., distal radius and tibia and aim at the quantitative assessment of structural cortical bone properties, e.g., Ct.Th and Ct.Po ${ }^{23}$. However, none of the existing diagnostic technologies can assess quantitative information about the cortical pore micro-morphology. Particularly, the transition from a normal, age- and gender specific pore size distribution to a pathologically altered one caused by large BMUs could not be assessed in vivo so far.

We have recently developed theoretical cortical bone backscatter model (CortBS) and an ultrasonic multi-angle 3-D acquisition and data processing scheme to assess viscoelastic and microstructural properties in cortical bone ${ }^{24}$. The method measures the frequency-dependent attenuation and backscatter coefficients $\alpha(f)$ and $B S C(f)$ at the tibia and retrieves the cortical pore size distribution Ct.Po.Dm.D by fitting a theoretical backscatter coefficient to the measured $B S C(f)$. In an ex-vivo study on bones from 19 human donors, pore size parameters describing the prevalence of large pores could be assessed. The combination of cortical thickness and CortBS parameters provided similar or better prediction accuracies of proximal femur stiffness and strength than $a B M D$.

In this cross-sectional study the CortBS method was applied for the first time in humans. Postmenopausal women with and without history of fragility fractures were included. We hypothesized that the viscoelastic and microstructural CortBS biomarkers can be assessed in-vivo and 
that they are associated with the occurrence of fragility fractures. The in-vivo short-term precision of the CortBS biomarkers was assessed and the fracture discrimination performance was compared with those of DXA and HR-pQCT.

\section{Results}

Study population. The patient characteristics are summarized in Table 1 . Twentynine subjects had at least one fragility fracture. Age and anthropometric data were not different between Fx and $\mathrm{nFx}$ groups (Table 1). More subjects with fragility fractures received antiresorptive treatment than subjects without fractures. The higher number of subjects treated with aromatase inhibitor was not significant $(p=0.06)$.

\begin{tabular}{|c|c|c|c|}
\hline & All Patients $(n=55)$ & $F x(n=29)$ & $\mathrm{nFx}(\mathrm{n}=26)$ \\
\hline Age (years) & $69.9 \pm 7.2$ & $69.2 \pm 7.5$ & $70.2 \pm 6.3$ \\
\hline Height (cm) & $164.2 \pm 7.6$ & $165.0 \pm 8.1$ & $163.1 \pm 7.2$ \\
\hline Weight (kg) & $62.1 \pm 8.3$ & $62.4 \pm 9.1$ & $61.0 \pm 6.7$ \\
\hline BMI $\left(\mathrm{kg} / \mathrm{m}^{2}\right)$ & $23.0 \pm 2.8$ & $22.9 \pm 2.8$ & $23.0 \pm 2.7$ \\
\hline \multicolumn{4}{|l|}{ Diseases } \\
\hline Diabetes & 3 & 2 & 1 \\
\hline Rheumatic diseases & 11 & 7 & 5 \\
\hline Other chronic inflammatory diseases & 4 & 2 & 2 \\
\hline \multicolumn{4}{|l|}{ Medication } \\
\hline Antiresorptive & 23 & $17^{*}$ & 6 \\
\hline Osteoanabolic & 5 & 4 & 1 \\
\hline Vitamin D & 54 & 28 & 26 \\
\hline SERM (Selective estrogen receptor modulator) & 3 & 1 & 2 \\
\hline Corticosteroid oral & 14 & 7 & 7 \\
\hline Corticosteroid inhaled & 3 & 2 & 1 \\
\hline Aromatase Inhibitor & 6 & 1 & 5 \\
\hline PPI & 4 & 1 & 3 \\
\hline Other Medications* & 25 & 15 & 10 \\
\hline
\end{tabular}

${ }^{*} p<0.05$

DXA: A valid vertebral spine T-score could not be assessed in 8 subjects due to severe degenerative changes in the lumbar spine. DXA parameters were associated with subject's height $\left(R^{2}=0.40\right)$, weight $\left(R^{2}=0.34\right)$, and almost independent of $B M I\left(R^{2}=0.23\right)$ and age $\left(R^{2}=0.14\right)$ (see Table A.1 in the Supplementary Materials). None of the $a B M D$ values and T-scores was significantly different between $\mathrm{Fx}$ and $\mathrm{nFx}$ groups (Table 2), but the difference of the lowest total T-scores between fractured and nonfractured groups almost reached the significance level $(p=0.06)$.

\begin{tabular}{|c|c|c|c|}
\hline Parameter & Range & Fx $(n=26)$ & $n F x(n=29)$ \\
\hline$a B M D_{\text {Femur(Total) }}\left[\mathrm{g} / \mathrm{cm}^{2}\right]$ & $0.668-1.004$ & $0.786 \pm 0.072$ & $0.808 \pm 0.076$ \\
\hline$a B M D_{\text {Femur }(\text { Neck) }}\left[\mathrm{g} / \mathrm{cm}^{2}\right]$ & $0.635-0.991$ & $0.793 \pm 0.081$ & $0.805 \pm 0.072$ \\
\hline$a B M D_{\text {Spine }}\left[\mathrm{g} / \mathrm{cm}^{2}\right]^{\mathrm{a}}$ & $0.651-1.242$ & $0.904 \pm 0.130^{\mathrm{b}}$ & $0.950 \pm 0.110^{c}$ \\
\hline T-score Femur & $-3.1-1.5$ & $-1.93 \pm 0.86$ & $-1.83 \pm 0.55$ \\
\hline T-scorespine ${ }^{a}$ & $-4.3-0.5$ & $-2.28 \pm 1.04^{b}$ & $-1.90 \pm 0.90^{c}$ \\
\hline T-score ${ }_{\text {Total }}{ }^{a}$ & $-4.3--1.4$ & $-2.41 \pm 0.72^{b}$ & $-2.14 \pm 0.66^{c}$ \\
\hline
\end{tabular}


HR-pQCT: Data from one subject could not be evaluated due to an apparent motion artefact. From the remaining 54 subjects, 81 structure and material properties were extracted. HR-pQCT parameters obtained from the scanner software were associated with subject's weight $\left(R^{2}=0.46\right)$, height $\left(R^{2}=0.42\right)$, age $\left(R^{2}=0.31\right)$ and almost independent of $B M I\left(R^{2}=0.20\right)$. Except for $B M I$, the associations of cortical parameters derived from the custom analysis with anthropometric data and age were generally lower (see supplementary Table A.1). None of the parameters derived from the scanner software was significantly different between fractured and non-fractured groups (Table 3 ). In contrast, most parameters describing the local distributions of porosity and pore diameter in the antero-medial region of interest were significantly different between both groups. The most prominent differences were observed for skewness $(p=0.004)$ and kurtosis $(p=0.004)$ of the pore size distribution Ct.Po.Dm.D evaluated in the full cross sections.

\begin{tabular}{|c|c|c|c|}
\hline \multicolumn{4}{|c|}{$\begin{array}{l}\text { Table } 3 \text { I HR-pQCT range, means and SDs of selected parameters in fractured anc } \\
\text { non-fractured groups. Significant differences are marked in bold letters. }\end{array}$} \\
\hline Bone Geometry & Range & $F x(n=29)$ & $n F x(n=25)$ \\
\hline$T t . A r\left[\mathrm{~mm}^{2}\right]$ & $313-536$ & $420 \pm 56$ & $407 \pm 47$ \\
\hline Ct.Pm $[\mathrm{mm}]$ & $72-100$ & $86 \pm 7$ & $84 \pm 5$ \\
\hline Ct.Ar $\left[\mathrm{mm}^{2}\right]$ & $170-318$ & $254 \pm 31$ & $252 \pm 40$ \\
\hline $\operatorname{Tb} . A r\left[\mathrm{~mm}^{2}\right]$ & $97-298$ & $170 \pm 47$ & $159 \pm 41$ \\
\hline Tb.Meta.Ar $\left[\mathrm{mm}^{2}\right]$ & $40-121$ & $69 \pm 19$ & $64 \pm 17$ \\
\hline Tb.Inn.Ar $\left[\mathrm{mm}^{2}\right]$ & $58-177$ & $101 \pm 28$ & $94 \pm 25$ \\
\hline \multicolumn{4}{|l|}{ Bone Density } \\
\hline Tt.vBMD [mg HA/cm $\left.\mathrm{cm}^{3}\right]$ & 439- 748 & $596 \pm 77$ & $611 \pm 65$ \\
\hline$T b . v B M D\left[\mathrm{mg} \mathrm{HA} / \mathrm{cm}^{3}\right]$ & $32-165$ & $77 \pm 30$ & $78 \pm 30$ \\
\hline Tb.Meta.vBMD [mg HA/ $\left.\mathrm{cm}^{3}\right]$ & $106-291$ & $184 \pm 51$ & $184 \pm 35$ \\
\hline Tb.Inn.vBMD [mg HA/cm $\left.{ }^{3}\right]$ & $-22-109$ & $4.3 \pm 20$ & $5.8 \pm 30$ \\
\hline Ct.vBMD [mg HA/ $\left./ \mathrm{cm}^{3}\right]$ & $826-1049$ & $930 \pm 53$ & $940 \pm 31$ \\
\hline \multicolumn{4}{|l|}{ Bone Structure } \\
\hline$B V / T V$ & $0.07-0.25$ & $0.13 \pm 0.04$ & $0.13 \pm 0.04$ \\
\hline $\operatorname{Tb} . N[1 / \mathrm{mm}]$ & $0.5-1.8$ & $1.12 \pm 0.29$ & $1.18 \pm 0.29$ \\
\hline Tb.Th $[\mathrm{mm}]$ & $0.19-0.36$ & $0.27 \pm 0.04$ & $0.27 \pm 0.03$ \\
\hline Tb.Sp $[\mathrm{mm}]$ & $0.56-2.05$ & $1.01 \pm 0.30$ & $0.94 \pm 0.29$ \\
\hline$T b .1 / N . S D[\mathrm{~mm}]$ & $0.18-1.31$ & $0.45 \pm 0.22$ & $0.39 \pm 0.18$ \\
\hline Ct.Th $[\mathrm{mm}]$ & $2.8-6.56$ & $4.96 \pm 0.57$ & $4.96 \pm 0.83$ \\
\hline Ct.Po [\%] & $0.4-8.2$ & $2.5 \pm 1.9$ & $2.0 \pm 1.0$ \\
\hline Ct.Po.Dm [mm] & $0.15-0.33$ & $0.21 \pm 0.04$ & $0.22 \pm 0.04$ \\
\hline \multicolumn{4}{|l|}{ Custom (ROI) } \\
\hline$C t . T h_{(\mathrm{ROI})}[\mathrm{mm}]$ & $1.0-4.2$ & $2.7 \pm 0.8$ & $2.7 \pm 0.6$ \\
\hline$C t . P O_{\mathrm{BH}(\mathrm{ROI})}[\%]$ & $1.1-11.1$ & $5.4 \pm 2.3$ & $4.5 \pm 2.2$ \\
\hline \multicolumn{4}{|l|}{ Cortical Porosity Distribution } \\
\hline Ct.Po.D $D_{\text {Mean(ROI) }}[\%]$ & $14.7-33.8$ & $26.0 \pm 4.6$ & $25.3 \pm 3.6$ \\
\hline Ct.Po.DsD(ROI) [\%] & $3.7-9.6$ & $6.0 \pm 1.2^{*}$ & $5.4 \pm 1.1$ \\
\hline Ct.Po.DVAR(ROI) $[\%]$ & $13.5-92.9$ & $37.5 \pm 16.2^{*}$ & $30.1 \pm 13.2^{*}$ \\
\hline$C t . P o . D_{\text {skewness(ROI) }}$ & $0.5-2.9$ & $1.0 \pm 0.4$ & $1.4 \pm 0.5$ \\
\hline$C t . P o . D_{\text {skewness(Full) }}$ & $0.48-2.93$ & $0.98 \pm 0.37^{*}$ & $1.35 \pm 0.51$ \\
\hline$C t . P o . D_{\text {kurtosis(ROI) }}$ & $3.1-17.2$ & $5.1 \pm 1.5$ & $7.3 \pm 3.4$ \\
\hline
\end{tabular}




\begin{tabular}{|c|c|c|c|}
\hline Ct.Po. $D_{\text {kurtosis(Full) }}$ & $3.1-17.2$ & $5.1 \pm 1.5^{*}$ & $7.3 \pm 3.4$ \\
\hline \multicolumn{4}{|c|}{ Cortical Pore Diameter Distribution } \\
\hline Ct.Po.Dm.D Mean(ROI) $[\mu \mathrm{m}]$ & $96-185$ & $128 \pm 20^{*}$ & $120 \pm 15$ \\
\hline Ct.Po.Dm.DSD(ROI) $[\mu \mathrm{m}]$ & $39-165$ & $83 \pm 28$ & $74 \pm 23$ \\
\hline Ct.Po.Dm.D Q90(ROI) $[\mu \mathrm{m}]$ & $153-417$ & $230 \pm 54 *$ & $205 \pm 35$ \\
\hline \multicolumn{4}{|c|}{ Cortical Bone BMD Distribution } \\
\hline$C t . B M D . D_{\text {kurtosis(Full) }}$ & $3.15-5.54$ & $3.49 \pm 0.48 *$ & $3.52 \pm 0.23$ \\
\hline
\end{tabular}

CortBS: An ultrasound compound image of the antero-medial region of the tibia of bone together with the normalized difference spectrum and representative backscatter and attenuation coefficients, and pore size distributions for subjects with and without fragility fractures are shown in Fig. 2 . The shortterm precision of the individual parameter estimations was in the range between 1.7 and $13.9 \%$ (Table 4). For 5 patients, the quality factor was below $77 \%$ and therefore, data were not analyzed. CortBS parameters were associated with subject's age $\left(R^{2}=0.45\right)$, height $\left(R^{2}=0.36\right)$, and marginally with weight $\left(R^{2}=0.25\right)$ and $B M I\left(R^{2}=0.22\right)$ (see supplementary Table A.1). Parameter ranges and differences between fractured and non-fractured groups are summarized in Table 3. Slope $C t$. $\alpha_{f}$ and intercept $C t . \alpha_{f}$ values of the attenuation coefficient were significantly different between fractured and non-fractured groups. The change of the width of the pore size distribution Ct.Po.Dm.DFWнM almost reached the significance level $(p=0.06)$.

\begin{tabular}{|c|c|c|c|c|}
\hline Parameter & Precision & Range & $F x(n=25)$ & $n F x(n=25)$ \\
\hline$C t . \alpha_{0}[\mathrm{~dB}]$ & $0.22(13.91)$ & $1.06-3.10$ & $2.34 \pm 0.40^{*}$ & $1.96 \pm 0.48$ \\
\hline$C t . \alpha_{f}[\mathrm{~dB} / \mathrm{mm}]$ & $0.02(15.29)$ & $0.01-0.32$ & $0.11 \pm 0.06 *$ & $0.16 \pm 0.06$ \\
\hline Ct.Po.Dm.D Peak $[\mu \mathrm{m}]$ & $2.51(8.47)$ & $24-52$ & $38.6 \pm 5.6$ & $36.6 \pm 7.1$ \\
\hline Ct.Po.Dm. $D_{\mathrm{Q} 10}[\mu \mathrm{m}]$ & $1.93(7.89)$ & $20-42$ & $29.9 \pm 4.7$ & $28.8 \pm 5.8$ \\
\hline Ct.Po.Dm. $D_{\mathrm{Q} 90}[\mu \mathrm{m}]$ & $3.28(8.64$ & $30-64$ & $48.7 \pm 7.1$ & $45.3 \pm 8.8$ \\
\hline Ct.Po.Dm.D $D_{\text {FWнM }}[\mu \mathrm{m}]$ & 1.43 (11.99) & $9.4-25.6$ & $16.5 \pm 3.5$ & $14.9 \pm 2.9$ \\
\hline Ct.Po.Dm.D $D_{\mathrm{FWHM}, \min }[\mu \mathrm{m}]$ & $2.09(8.53)$ & $20.0-42.8$ & $31.1 \pm 5.0$ & $29.5 \pm 5.8$ \\
\hline Ct.Po.Dm.D $D_{\mathrm{FWHM}, \max }[\mu \mathrm{m}]$ & $3.15(8.62)$ & $29.4-62.8$ & $47.6 \pm 6.8$ & $44.4 \pm 8.6$ \\
\hline$C t . B S C_{\text {mean }}[\mathrm{dB}]$ & $0.52(2.97)$ & $-18.7--12.1$ & $-15.7 \pm 1.5$ & $-15.6 \pm 1.7$ \\
\hline Offset $[\mathrm{dB}]$ & $0.46(10.82)$ & $-10.1--2.67$ & $-5.9 \pm 1.6$ & $-6.0 \pm 1.9$ \\
\hline
\end{tabular}

Fragility Fracture Discrimination: The results of discrimination performance analyses are summarized in Fig. 4 and Table 5. The DXA based T-score values reached the lowest discrimination performance for all types of fragility fractures $(0.51 \leq A \cup C \leq 0.55)$. Incorporating subject's weight and height could slightly improve the discrimination performance for vertebral and other fractures. Among all HR-pQCT parameters, those describing the shape distributions of porosity and pore diameter were the most predictive ones. Distinct parameter combinations provided good discrimination models for vertebral, non-vertebral, and all fragility fractures $(0.65 \leq \mathrm{AUC} \leq 0.73)$. Age and anthropometric information could not further improve the discrimination models. CortBS parameters provided very good discrimination models for all types of fragility fractures $(0.65 \leq \mathrm{AUC} \leq 0.72)$. While for non-vertebral fractures only attenuation parameters $\left(C t . \alpha_{0}\right.$ and $\left.C t . \alpha_{f}\right)$ were selected, vertebral and all fractures were discriminated by a combination of attenuation and pore size distribution parameters. The 
discrimination performance was further improved by weight and height information $(0.69 \leq \mathrm{AUC} \leq 0.75)$.

a

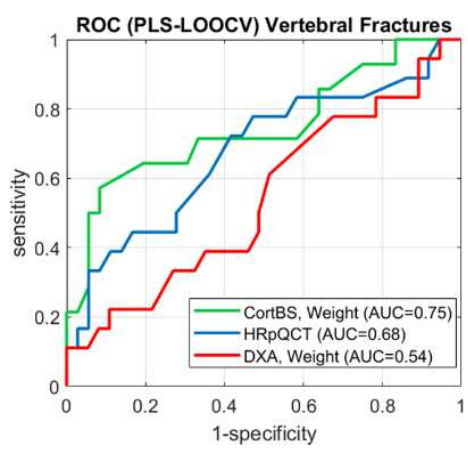

b

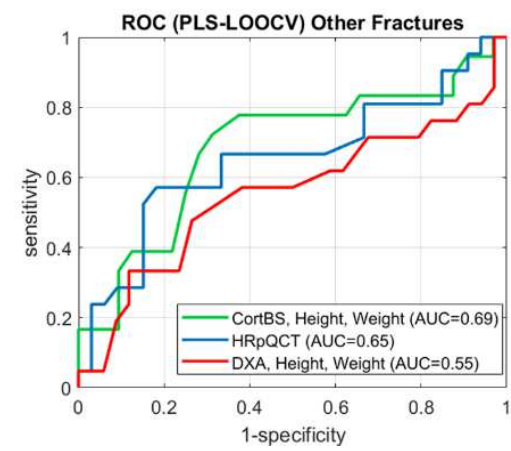

C

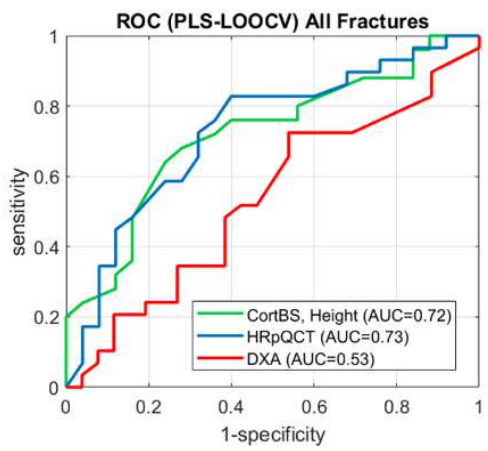

Fig. 4 Fragility fracture discrimination performance of DXA, HR-pQCT and CortBS for vertebral fractures (a) other fractures (b), and all fractures (c). If anthropometric information improved the discrimination model, these ROC curves are shown.

\begin{tabular}{|c|c|c|c|c|c|c|}
\hline & Sensitivity & Specificity & $A \cup C$ & Accuracy & OR $(95 \% \mathrm{Cl})$ & Variables \\
\hline \multicolumn{7}{|c|}{ Vertebral Fractures } \\
\hline DXA & 0.18 & 0.97 & 0.51 & 0.79 & $7.9(5.3-10.3)$ & $\begin{array}{l}\text { T-score } \\
\text { T-scomur } \\
\text {-scoresine }\end{array}$ \\
\hline$D X A+A P$ & 0.11 & 0.94 & 0.54 & 0.67 & $2.2(0.1-4.2)$ & $\begin{array}{l}\text { T-scorefemur } \\
\text { Weight }\end{array}$ \\
\hline HR-pQCT & 0.27 & 0.92 & 0.68 & 0.70 & $4.2(2.7-5.8)$ & 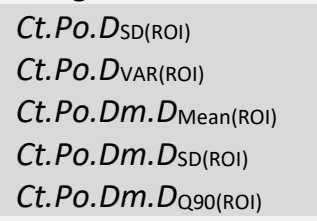 \\
\hline CortBS & 0.43 & 0.92 & 0.72 & 0.78 & $8.2(6.7-9.8)$ & $\begin{array}{l}\text { Ct. } \alpha_{o}, C t . \alpha_{f} \\
\text { Ct.Po.Dm.DFWHM } \\
\text { Ct.Po.Dm.DFWHM-Max } \\
\text { Ct.Po.Dm.DQ90 }\end{array}$ \\
\hline CortBS + AP & 0.50 & 0.94 & 0.75 & 0.82 & $17.0(15.2-18.8)$ & $\begin{array}{l}\text { Ct. } \alpha_{0}, C t . \alpha_{f} \\
\text { Ct.Po.Dm.DFWHM } \\
\text { Ct.Po.Dm.Dogo } \\
\text { Weight }\end{array}$ \\
\hline \multicolumn{7}{|c|}{ Other Fractures } \\
\hline DXA & 0.12 & 0.87 & 0.55 & 0.60 & $0.9(-0.9-2.7)$ & T-score Total \\
\hline$D X A+A P$ & 0.33 & 0.85 & 0.55 & 0.65 & $2.9(1.6-4.2)$ & $\begin{array}{l}\text { T-score } \text { Femur } \\
\text { Height, Weight }\end{array}$ \\
\hline HR-pQCT & 0.48 & 0.85 & 0.65 & 0.70 & $5.1(3.8-6.4)$ & $\begin{array}{l}\text { Ct.POBH(ROI) } \\
\text { Ct.Po.Dskewnes(ROI) } \\
\text { Ct.PO.Dn(ROI) } \\
\text { Ct.POBH(Full) } \\
\text { Ct.Po.Dskewnes(Full) } \\
\text { Ct.Po.D } D_{\text {kurtosis(Full) }} \\
\text { T.Tb.Th(Full) }\end{array}$ \\
\hline CortBS & 0.39 & 0.81 & 0.65 & 0.66 & $2.76(1.5-4.1)$ & Ct. $\alpha_{0}, C t . \alpha_{\mathrm{f}}$ \\
\hline
\end{tabular}




\begin{tabular}{|c|c|c|c|c|c|c|}
\hline CortBS + AP & 0.39 & 0.88 & 0.69 & 0.70 & $4.45(3.0-5.9)$ & $\begin{array}{l}\text { Ct. } \alpha_{0}, \text { Ct. } \alpha_{f} \\
\text { Height, Weight }\end{array}$ \\
\hline \multicolumn{7}{|c|}{ All Fractures } \\
\hline DXA & 0.52 & 0.58 & 0.53 & 0.54 & $1.5(0.3-2.5)$ & T-score Total \\
\hline HR-pQCT & 0.83 & 0.64 & 0.73 & 0.74 & $8.5(7.3-9.8)$ & $\begin{array}{l}\text { Ct.Po. } D_{\mathrm{Q} 90(\mathrm{ROI})} \\
\text { Ct.Po. } D_{\text {skewness(Full) }}\end{array}$ \\
\hline CortBS & 0.68 & 0.64 & 0.69 & 0.66 & $3.8(2.6-4.9)$ & $\begin{array}{l}\text { Ct. } \alpha_{0}, C t . \alpha_{f} \\
\text { Ct.PoDm. } D_{Q 10} \\
\text { Ct.PoDm. } D_{Q 90} \\
\text { Ct.PoDm.D Peak } \\
\text { Ct.PoDm.DFWHM-Min } \\
\text { Ct.PoDm.DFWHM-Max }\end{array}$ \\
\hline CortBS + AP & 0.72 & 0.64 & 0.72 & 0.68 & $4.6(3.4-5.8)$ & $\begin{array}{l}\text { Ct. } \alpha_{0}, \text { Ct. } \alpha_{f} \\
\text { Height }\end{array}$ \\
\hline
\end{tabular}

Associations between HR-pQCT and CortBS parameters. Attenuation was not associated with bone geometry except for one weak correlation between Ct. $\alpha_{0}$ and Ct.Th (see Table A.2 in the Supplementary Materials). Multiple univariate associations were found for attenuation and Ct.Po.Dm.D parameters with bone density, structure, and porosity and pore diameter distributions. Most HR-pQCT parameters could be predicted with weak to moderate accuracy $\left(0.13 \leq R^{2} \leq 0.50\right)$ by combinations of CortBS parameters.

Ultrasound-based BMD prediction: Figure 5 shows the prediction of $a B M D$ from CortBS parameters using multivariate PLS models. Although significant, the correlations were weak to moderate $\left(0.25 \leq R^{2} \leq 0.34\right)$.

a

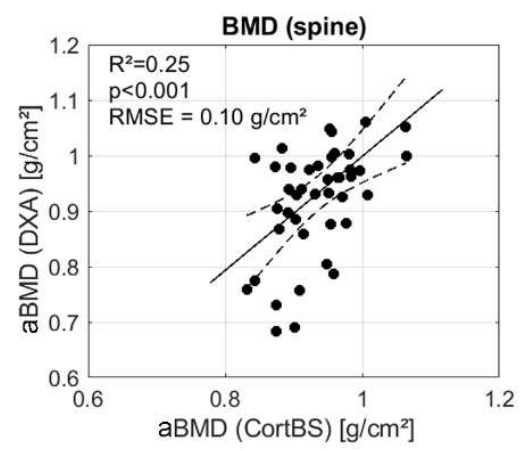

b

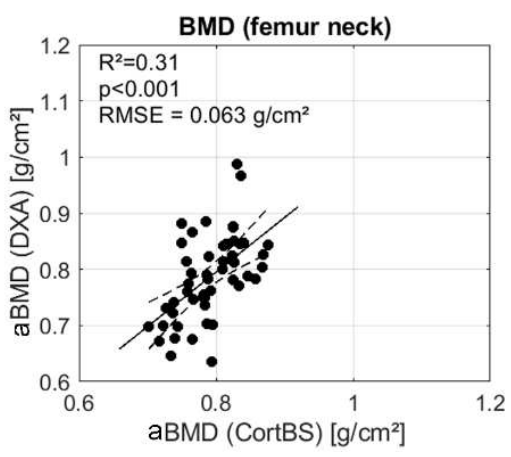

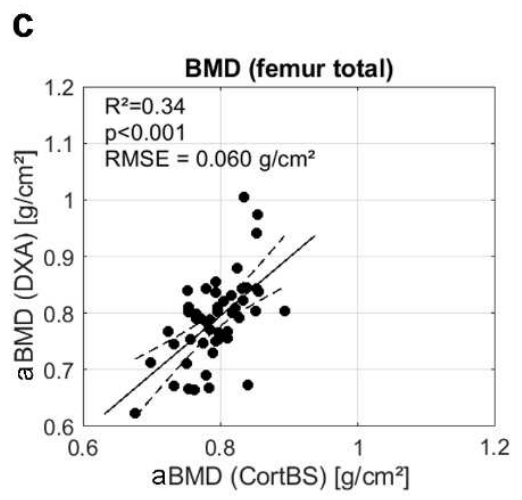

Fig. 5 Estimation of aBMD at spine (a), femur neck (b) and total proximal femur (c) from ultrasound backscatter parameters using PLS regression.

\section{Discussion}

The diagnosis of osteoporosis based on the assessment of structural deterioration of the porous micromorphology has been prevented by the limitations of currently available diagnostic technologies 7. The established diagnosis of OP based on $a B M D$ captures the relative bone loss of an individual compared to a mean value of young reference population, but not the individual bone loss caused by impaired bone remodeling. Thereby, people with non-pathologically decreased T-scores, but at risk for fragility fractures are currently undiagnosed until the fracture occurs ${ }^{8}$. Alternative diagnostic modalities provide $\mathrm{x}$-ray radiation free $B M D$ and T-score surrogates ${ }^{25-28}$, but do not overcome the principal lack of sensitivity of $B M D$ to the causal microstructural and tissue deteriorations caused by OP. To date, HR-pQCT is the most sophisticated in-vivo imaging modality for the microstructural analysis of bone. It can resolve pathologically enlarged cortical pores, but the spatial resolution is not 
sufficient to resolve the normal cortical pore micromorphology ${ }^{18}$. In this work, we have applied for the first time in humans a novel ultrasound technology that that provides non-invasively and without ionizing radiation quantitative information about the heterogeneous pore size distribution together with viscoelastic bone properties in cortical bone at the tibia midshaft. The fracture discrimination performance of the novel CortBS technology was benchmarked against conventional DXA-based diagnosis and state-of-the-art x-ray computed tomography (HR-pQCT).

The anteromedial tibia is a favorable site for the assessment of systemic viscoelastic and structural bone tissue deteriorations leading to fragility fractures. The standard locations for DXA measurements (i.e., L1-L4 lumbar spine, and hip and forearm) were initially selected because morbidity from fractures at these locations is high ${ }^{7}$. However, metal implants, previous fractures, scoliosis, osteosclerosis, and aortic calcifications render $a B M D$ estimations at these sites inaccurate or even impossible ${ }^{29}$. The most standardized HR-pQCT measurement site is the distal tibia ${ }^{30}$. In an ex-vivo study, hip failure load has been reported to be associated with low $V B M D$ and microstructural alterations measured at this site ${ }^{31}$. However, towards the epiphyses cortical bone becomes thinner and is increasingly replaced by a trabecular core. Therefore, parameters assessed in this region are susceptible to both positioning errors and inter-subject anatomical differences. In this study, we have used the tibia midshaft region for the following reasons. First, the midshaft contains predominantly cortical bone. Second, the cortical thickness in the antero-medial measurement midshaft region is relatively invariant with respect to the long-axis position and approximately 2 to 3 times larger ${ }^{32}$ than reference values reported for the proximal tibia ${ }^{33}$. Thereby, the tibia midshaft provides a much larger and anatomically more invariant tissue volume for cortical bone microstructural characterization than the distal shaft. Recent ex-vivo studies provided evidence that structural deterioration at this measurement site is associated with reduced proximal femur strength ${ }^{32}$ and that the biomarkers assessed by CortBS combined with Ct.Th provide superior predictions of proximal femur stiffness and strength compared to $a B M D^{24}$. Ultrasound can be transmitted most easily to and along bone at the facies medialis of the tibia midshaft, where the periosteum is covered by a thin layer of soft tissue only. Already in 1995, Foldes et al. ${ }^{34}$ suggested speed of sound measured by axial transmission at the tibia midshaft as an independent predictor of fracture risk in women with non-osteoporotic bone mineral density ${ }^{34}$. Since then, various novel bone QUS techniques have targeted this site for the measurement of Ct.Th, Ct.Po ${ }^{35,36}$, and speed of sound ${ }^{37}$. This study confirmed that i) pore architecture and viscoelastic properties of the cortical tibia midshaft decay with age ${ }^{12}$ and ii) are associated with bone fragility. In line with the well-known microstructural deteriorations induced by OP, both HR-pQCT and CortBS revealed predominantly features describing the asymmetry of the cortical pore size distribution rather parameters describing the mean pore-tissue volume fraction as factors associated with fragility fractures. Moreover, frequency-dependent ultrasound attenuation, which is determined by both structural and viscoelastic tissue properties ${ }^{24}$ was found to be significantly altered in subjects with fragility fractures.

Discrimination performance: The results of this pilot study suggest a superior discrimination performance of the ultrasonic cortical backscatter measurement $(0.69 \leq A \cup C \leq 0.75)$ compared to DXA $(0.53 \leq \mathrm{AUC} \leq 0.55)$ and a similar performance compared to HR-pQCT $(0.68 \leq \mathrm{AUC} \leq 0.73)$. The two attenuation parameters $C t . \alpha_{0}$ and $C t . \alpha_{f}$ were the strongest predictors for all types of fragility fractures. Together with subject's height and weight, cortical bone attenuation provided the best discrimination performance for non-vertebral fractures ( $A \cup C=0.69$ ). Subject's height is a known risk factor for nonvertebral fractures ${ }^{38}$, which has been partly linked thinner and more porous cortices in taller women, as measured at the distal tibia by first-generation HR-pQCT ${ }^{39}$. While $\mathrm{Ct}$. Th at the tibia mid-shaft was not a predictive variable in our study, the higher porosity together with other variations of the porosity and pore size distributions were observed both in HR-pQCT and CortBS measurements. 
For vertebral fractures, width and $90-\%$ quantile values of the pore diameter distribution were additional predictor variables, while subjects weight remained the only anthropometric factor (AUC = 0.75). This finding is in agreement a previous report suggesting risk factors, e.g. physical weakness, poor health, and weight loss as risk factors for vertebral, but not for non-vertebral fractures ${ }^{40}$.

Our AUC values were lower for DXA and comparable for QUS parameters than those reported in another study, in which cortical thickness and porosity were estimated from axial transmission ultrasound ${ }^{41}$. While in that study on 201 postmenopausal women $C t$. Th in was found to be discriminant for hip fractures only ( $A \cup C=0.72$ ), Ct. Po was discriminant for all fractures ( $A \cup C=0.71$ ), for vertebral $(A \cup C=0.84)$ and wrist fractures $(A \cup C=0.71)$.

Several bone QUS technologies have been used in the past to measure cortical or cancellous bone sites, and at least some of them have demonstrated the potential to predict fracture risk with an equivalent efficiency compared to X-ray densitometry techniques ${ }^{21,42}$. Although ultrasound wave propagation is governed by the structural and material properties of the propagation medium, none of the currently available clinical devices provide any direct measurement of stiffness, strength, or tissue quality. Instead, they provide bone density, stiffness or quality surrogate markers derived from empirical correlations of acoustic properties (e.g., speed of sound SOS and broadband ultrasound attenuation BUA ${ }^{21}$, travel time delays ${ }^{26,43}$, or the shape of the backscatter spectrum ${ }^{28}$ with $a B M D$. For example, Adami et al. ${ }^{44}$ used T-scores derived from Radiofrequency Echographic Multi Spectrometry (REMS) in comparison with DXA-based T-scores for the discrimination of women with and without fractures the identification of patients at risk for incident osteoporotic fractures. In this prospective study on 1516 Caucasian women (age: 30-90 years) reported similar prediction performance for DXA and QUS based T-scores. A model-based measurement of Ct.Th and Ct.Po in radius and tibia bones has been achieved for the first time with the bidirectional axial transmission technology by means of multimode waveguide dispersion analysis ${ }^{35,45}$. The method considers variations of porosity as a major source of variations of cortical bone elasticity, sound velocity and fracture toughness in postmenopausal women ${ }^{46-48}$. Results of a first validation study in postmenopausal women confirmed a comparable fracture discrimination performance of the BDAT variables as $a B M D$ for both vertebral and peripheral fractures ${ }^{41}$. However, axial transmission measurements do not provide direct image-guidance and are restricted to patients with low BMI.

CortBS reflects viscoelastic and microstructural deteriorations of cortical bone, which are causally linked to natural aging process and the development of osteoporosis ${ }^{12}$. The crucial role of the porous microarchitecture, particularly the prevalence of large pores as biomarker for reduced bone strength

${ }^{32}$ was also confirmed in the HR-pQCT analysis, which revealed the asymmetry of the porosity distribution, but not the total porosity as a fracture discriminating tissue property. In contrast to that ex-vivo study, which included bone from both male and female donors, $C t$. Th was not found to be a fracture discriminating biomarker in our study.

Limitations. This pilot study has several limitations. First, the cohort size was small and restricted to post-menopausal women with T-scores below -1 . The included subjects had diverse fracture and medication histories as well as various co-morbidities. However, the selected cohort resembles the population that is i) most vulnerable for fragility fractures and ii) mostly undertreated based on the $B M D$ diagnosis. Despite these limitations, a strong discrimination performance was achieved, which needs to be confirmed in larger studies covering a larger age range, both gender, larger BMI ranges, and T-scores above -1 . Second, the cross-sectional study design did not allow to assess fracture risk. Future prospective studies should therefore evaluate the potential of CortBS parameters to identify people at risk and to assess the individual fracture risk. Second, no real-time assessment of the CortBS measurement quality was possible in this study, which led to the exclusion of data from 5 subjects 
during the post-hoc data analysis. For clinical applications, the data quality assessment needs to be incorporated into the measurement, providing real-time feedback to the operator and the possibility to repeat the measurement, until an appropriate data quality is achieved.

\section{Conclusion}

CortBS is the first quantitative bone imaging modality that can quantify viscoelastic and microstructural tissue deteriorations in cortical bone, which occur during normal aging and the development of osteoporosis. CortBS discriminates fragility fractures in postmenopausal women better than, and independent of $a B M D$. It could be used as a portable, low-cost, non-ionizing, and widely applicable screening tool to identify people at risk, particularly in the population with low bone mass. A widespread application of the method is anticipated to enable an early identification of people at increased risk, a timely initiation of preventive therapies, and subsequently to a reduction of the prevalence of fragility fractures in people with metabolic bone diseases.

\section{Methods}

Research participants. For this cross-sectional study, 55 female subjects (age $\geq 55$ years) who have been referred to the Center for Muscle and Bone Research for a clinically indicated DXA bone density measurement were recruited. Height, weight, age, medical history regarding diseases affecting bone health, chronical diseases, fracture status with differentiation regarding adequate/inadequate trauma, medications negatively affecting bone health, as well as osteoprotective and osteoanabolic medications were assessed. To reflect the distribution of fracture rate with respect to $B M D$ in postmenopausal women (Fig. 1), the patient recruitment was stratified into three groups according to the results of the DXA measurement (lowest T-Score of lumbar spine and proximal femur) and fracture status, i.e., OP: osteoporosis (T-Score $\leq-2.5$ ); OPE-Fx: osteopenia (T-Score between -1 and -2.5 ) and prevalent fragility fracture); OPE-nFx: osteopenia (T-Score between -1 and -2.5 ) without prevalent fragility fracture. Exclusion criteria were i) $\mathrm{BMI}>30$; (ii) presence of metal implants or edema at the lower extremity; (iii) no allowance for X-ray exposure; or iv) the inability to understand the nature of the study and follow the instructions. In addition to the measurements on patients, repeated ultrasound readings were performed on three healthy volunteers. The study was registered in the German Clinical Trial Register (DRKS00022217) and was approved by the local ethics committee of the Charité - University Hospital Berlin (reference number: EA4/068/19) and the German Federal Office for Radiation Protection (reference number: Z5-22464/2019-090-G). All participants provided their informed written consent prior to participation.

DXA bone densitometry. DXA (Lunar Prodigy Advance EnCore Software v13.4 or Lunar iDXA EnCore Software v 16.1, GE Medical Systems, Wisconsin, USA) lumbar spine (L1-4) and proximal femur scans were performed as part of the clinical routine examination according to the standard GE LUNAR operator manual. The leg (left or right side) with the lowest $a B M D$ at the proximal femur was defined as index leg for subsequent ultrasound and HR-pQCT readings. $a B M D$ values were assessed at the

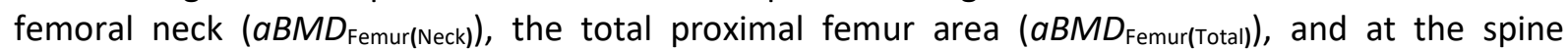
$\left(a B M D_{\text {spine }}\right)$. T-score were calculated, whereas the minimum scores at femur (T-score Femur $_{\text {) }}$, spine Tscore $_{\text {spine, }}$ and the lowest value of both anatomical regions ( $T$-score Total $)$ were used for further analyses. If the T-score from one region could not be assessed, the other one was defined as T-score Total. $_{\text {. }}$

Cortical Backscatter (CortBS). The measurement principle has been described in detail previously ${ }^{24}$. Briefly, a medical ultrasound scanner SonixTOUCH equipped with a SonixDAQ single-channel data acquisition system and a 4DL14-5/38 3-D linear array transducer (Ultrasonix, Richmond, Canada) was used. The system was controlled through a custom-developed user interface. Measurements were performed at the central antero-medial tibia region. The tibia length $\left(\mathrm{L}_{\mathrm{T} i \mathrm{bia}}\right)$ was assessed as the distance between the medial knee joint cleft and the medial malleolus. Both landmarks were palpated 
manually. Between these two points, the level of $50 \% \mathrm{~L}_{\text {Tibia }}$ was marked with a skin marker pencil. The ultrasound transducer was coupled to the skin at this position using an ultrasound coupling pad (aquaflex ${ }^{\circledast}$, Parker Laboratories, Inc., Fairfield, NJ). Conventional B-mode images were used to position the probe such that a cross-sectional image of the periosteal tibia bone interface appeared in the center of the image. The probe was then manually tilted until the bone surface was approximately normal to the sound beam direction and the focus position $F_{z}$ was adjusted to be approximately $1 \mathrm{~mm}$ below the periosteal bone surface (Fig. 2a). For the measurement, a compound B-mode volume scan sequence was used, which i) scanned a focused beam produced by a 16-element aperture across the array from element position 1 to 128 with an increment of 1 , ii) repeated the scan three times with different beam steering angles $\left(-10^{\circ}, 0^{\circ}, 10^{\circ}\right)$, and iii) repeated this scan with variable sweep motor positions and respective array tilt angles in the direction perpendicular to the B-mode image plane between $\pm 7^{\circ}$ with an increment of $1^{\circ}$ (Fig. 2a). For each transmit beam, pre-beamformed pulse-echo data were acquired from all 128 elements of the probe. The scan duration was less than 3 seconds. The signal analysis consists of 1 ) reconstruction of beamformed compound images for all sweep motor positions, i.e., spatial compounding of all three beam steering angles, (Fig. 2b); 2) manual selection of a region of interest covering the bone region to be analyzed (Fig. 2b); 3 ) automatic detection of the periosteal bone surface within the entire image volume (Fig. 2 b); 4) calculation of local beam inclinations, inclination-corrected surface reflection spectrum and an inclination-controlled depthdependent normalized difference spectrum NDS from the pre-beamformed channel data (Fig. 2c); 5), estimation of the frequency-dependent cortical bone attenuation and backscatter coefficients $\alpha(f)$ and $B S C(f)$, respectively (Figs. 2d-e); and 6) the estimation of the cortical pore diameter distribution Ct.Po.Dm.D (Fig. 2f). The latter is obtained by minimizing the error between the measured and theoretical BSCs, which are modelled from arbitrary pore size distributions. Except for step 2), all analysis steps were processed fully automatically. A quality parameter, which provides a relative measure of usable data within the selected ROI (i.e., data were not discarded by inclination, signallevel, and other thresholds) was used as an objective criterion to either accept or reject a measurement. Based on repeated measurement with variable probe tilt, a quality score threshold of $77 \%$ was found to produce reproducible results (data not shown). From $C t . \alpha(f)$, slope and intercept values $C t . \alpha_{f}$ and $C t . \alpha_{o}$ were obtained by linear regression (Fig. $2 d$ ). Characteristic parameters describing the asymmetric pore diameter distribution (i.e., $10 \%$ and $90 \%$ quantiles; width, minimum and maximum crossing points of full-width half-maximum (FWHM) values; and peak position were derived. 
a

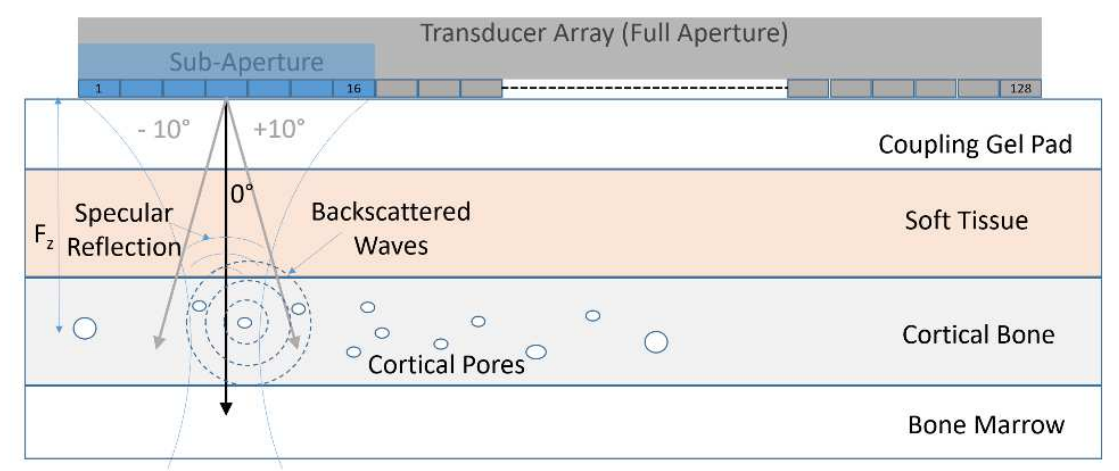

b

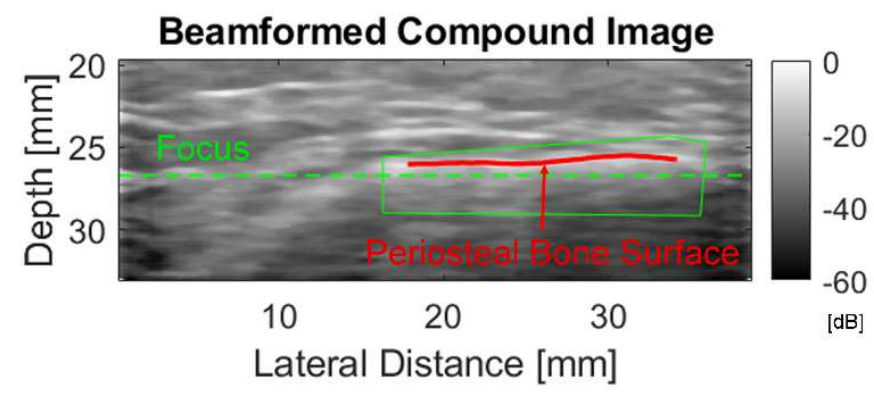

C

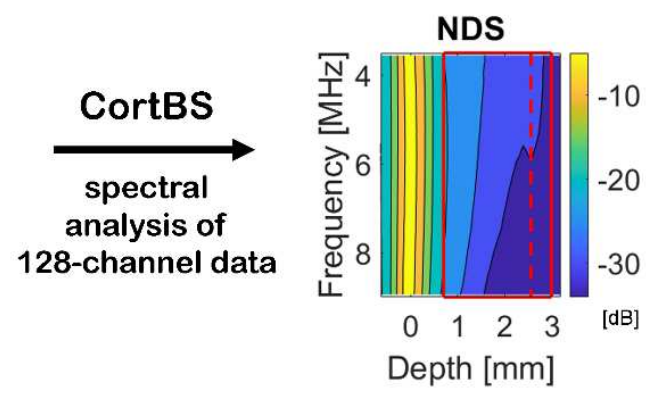

f

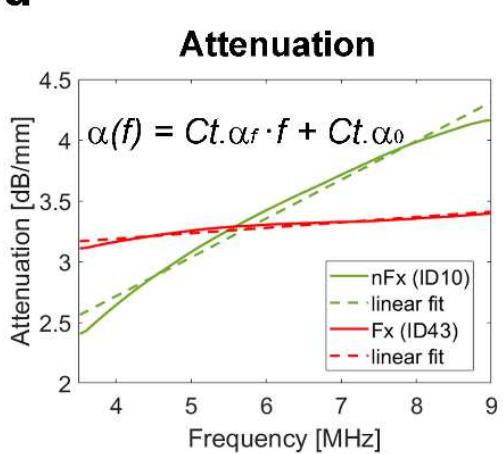

\section{e}

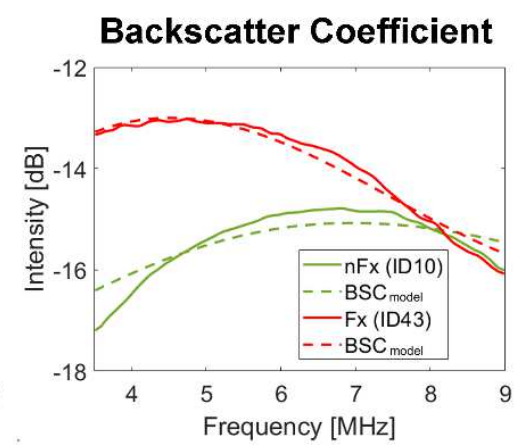

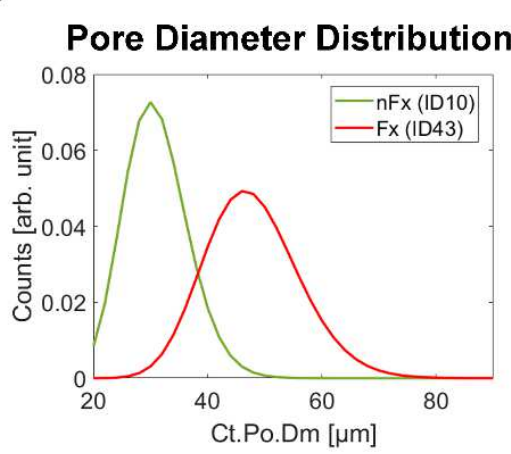

Fig. 2 Schematic drawing of the CortBS method (a). A focused beam generated by a 16-element sub-aperture of the 128element transducer array is scanned and steered across the bone. The focus depth $F_{z}$ is positioned approximately $1 \mathrm{~mm}$ below the bone surface. Pulse-echo signals are recorded simultaneously with all 128 channels. The reconstructed compound B-mode compound image (b) shows the antero-medial cross-section of the tibia midshaft (green dashed line: focus position; green line: manually selected ROI; red line: detected periosteal interface within ROI). The reconstructed 3D-bone surface (red line) is used to calculate a depth-dependent spectrogram. Spectra arising from specular reflections at the bone surface are used for normalization. From the normalized depth-dependent backscatter spectrum (NDS) (c), the depth and frequency ranges of 1$3 \mathrm{~mm}$ and 4-9 MHz, respectively, are used to derive the attenuation and backscatter coefficients $\alpha(f)$ (d) and BSC(f) (e). By fitting model-based backscatter coefficients ${ }^{24}$ to the measured $B S C(f)$, the pore diameter distribution is estimated (f). Panels (e-f) show representative $\alpha(f)$, and BSC(f), and Ct.Po.Dm.D data for one subject with (ID43; T-score Total $\left._{1}=-1.7\right)$ and one without fragility fractures (ID10; T-score Total $=-3.5)$.

CortBS short-term precision. The short-term precision was evaluated according to ${ }^{49}$ by performing 10 repeated measurements with repositioning between each measurement on three healthy volunteers. Absolute and relative precision values were calculated using Eq. (4a) and (5) in ${ }^{49}$, respectively.

High-resolution peripheral computed tomography (HR-pQCT). Immediately following the CortBS measurement, a site matched HR-pQCT scan was performed (XtremeCT II, Scanco Medical AG, Bassersdorf, Switzerland). Subjects were seated in a comfortable, height-adjustable chair. The lower leg of the subjects was positioned carefully in a carbon-fiber cast and fixated in the gantry. Subjects 
were instructed to sit as still as possible and do not talk or move to avoid motion artefacts. The gantry was moved into the scanner until the skin mark and the laser position indicator were aligned. A total scan length of $10.2 \mathrm{~mm}$ in the axial direction divided into 168 cross-sectional images was measured with an isotropic voxel size of $60.7 \mu \mathrm{m}$ with a scan time of 2 minutes. The total effective dose was less than $5 \mathrm{mSv}$ per scan. A representative reconstructed cross-sectional image is shown in Fig. 3a. Cortical and trabecular properties of the tibia were evaluated using the "3D Density and Structure Analysis" software of the scanner as described elsewhere ${ }^{30}$. Moreover, cortical properties of i) the entire tibia cross-section (Full) and ii) a manually selected antero-medial region of interest (ROI, see Fig. 3) were evaluated using a custom protocol adapted from ${ }^{18}$. This analysis estimates cortical porosity $C$ Ct. $P O_{B H}$ using the algorithm proposed by Burghardt ${ }^{50}$, cortical thickness Ct.Th, pore density Ct.Po.Dn, and distributions of porosity Ct.Po.D, pore diameter Ct.Po.Dm, and bone mineral density Ct.BMD.D ${ }^{18}$. From these distributions, characteristic properties, i.e., mean, standard deviation variance, skewness, kurtosis, as well as 10 and $90 \%$ quantile values were derived.
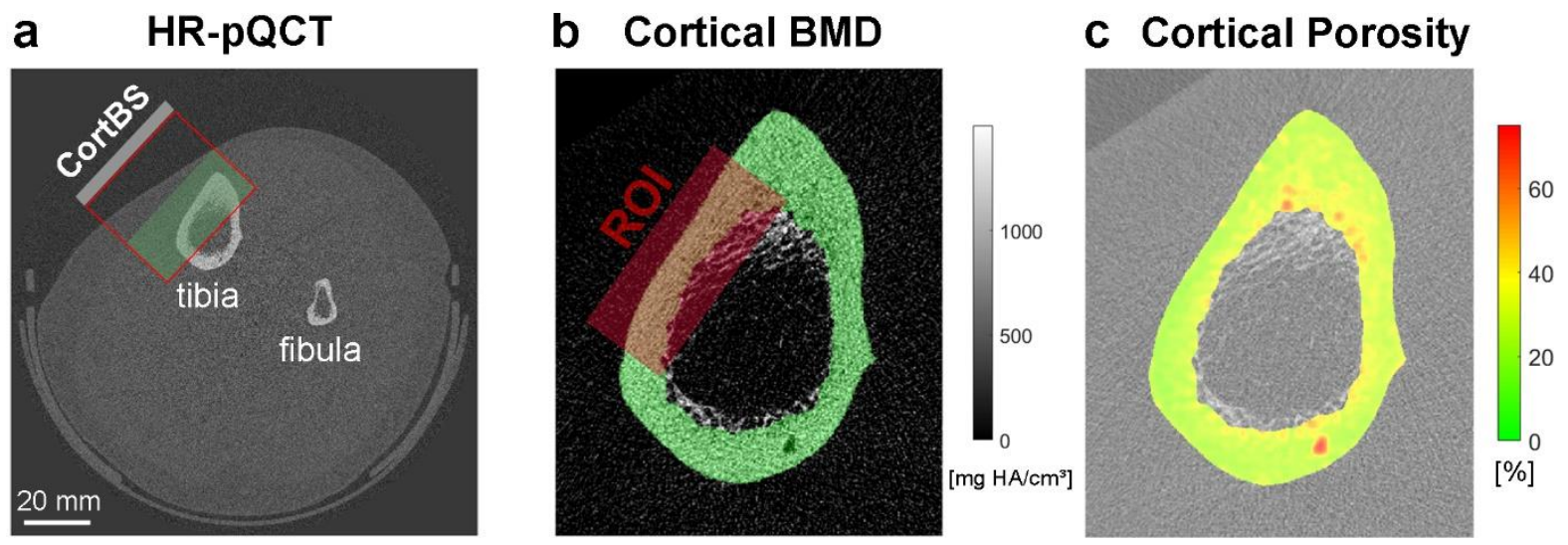

Fig. 3 Representative cross-sectional HR-pQCT image with the overlaid CortBS measurement region (a). The box shaded in green is the image region shown in Fig. $2 b$. The full tibia cross-section and the site-matched CortBS measurement region were analyzed (b). Most of the pores in the cortical bone (marked in green) are unresolved, but result in local fluctuations of the voxel values. Pores larger than $\sim 90 \mu \mathrm{m}$ are resolved. The calculation of a local porosity map (c) adapted from lori et al. ${ }^{18}$.

Statistics. Continuous variables are presented as means and standard deviations (SD). Nonparametric tests were used. Wilcoxon rank sum tests were performed to determine whether parameters were significantly different between the non-fractured and fractured groups. The correlation between ultrasound and $x$-ray parameters was assessed using Spearman's rank sum correlation coefficient $\rho$. To evaluate the correlation of multiple QUS parameters with HR-pQCT and DXA parameters, partial least squares (PLS) regression with three-fold cross validation was used. The coefficient of correlation $\mathrm{R}^{2}$ and root mean square error (RMSE) between the predicted QUS based parameter and those measured by HR-pQCT or DXA were computed. The fragility fracture discrimination performance of CortBS, HR-pQCT, and DXA was assessed by means of multivariate PLS discrimination analyses with Leave-One-Out Cross-Validation (PLS-LOOCV) using the libPLS library ${ }^{51}$. For variable selection, a Subwindow Permutation Analysis (SPA) using 10.000 Monte Carlo samplings was repeated until a stable set of significant model variables was found. To avoid overfitting, the final models were created using 3 PLS components. Different discrimination models were developed to predict vertebral, other, and all fragility fractures from DXA based T-scores, HR-pQCT, or CortBS parameters and for combinations with subject's anthropometric data (weight, height, BMI) and age. The area under the curve (AUC) of the receiver operation characteristics (ROC), accuracy, sensitivity, sensitivity, and Odds Ratio (OR) with $95 \%$ Confidence Intervals (Cl) were calculated. Except for the PLS-LOOC and SPA 
analyses, all statistical tests were performed using the Statistics Toolbox of Matlab R2019b (MathWorks, Natick, MA). Statistical results were considered significant for $p$-values $<0.05$.

\section{References}

1 Feng, X. \& McDonald, J. M. Disorders of bone remodeling. Annu Rev Pathol 6, 121-145, doi:10.1146/annurev-pathol-011110-130203 (2011).

2 Borgstrom, F. et al. Fragility fractures in Europe: burden, management and opportunities. Arch Osteoporos 15, 59, doi:10.1007/s11657-020-0706-y (2020).

3 Cawthon, P. M. Gender Differences in Osteoporosis and Fractures. Clin Orthop Relat R 469, 1900-1905, doi:10.1007/s11999-011-1780-7 (2011).

4 Dhanwal, D. K., Dennison, E. M., Harvey, N. C. \& Cooper, C. Epidemiology of hip fracture: Worldwide geographic variation. Indian J Orthop 45, 15-22, doi:10.4103/0019-5413.73656 (2011).

5 Kanis, J. A. et al. European guidance for the diagnosis and management of osteoporosis in postmenopausal women. Osteoporos Int 30, 3-44, doi:10.1007/s00198-018-4704-5 (2019).

6 Rendl, S., Lapa, C., Blumel, C., Bundschuh, R. A. \& Schneider, P. Decision making for osteoporotic treatment using FRAX or DVO risk algorithms in a clinical setting. $J$ Musculoskelet Neuronal Interact 13, 339-345 (2013).

7 Choksi, P., Jepsen, K. J. \& Clines, G. A. The challenges of diagnosing osteoporosis and the limitations of currently available tools. Clin Diabetes Endocrinol 4, 12, doi:10.1186/s40842018-0062-7 (2018).

8 Hernlund, E. et al. Osteoporosis in the European Union: medical management, epidemiology and economic burden. A report prepared in collaboration with the International Osteoporosis Foundation (IOF) and the European Federation of Pharmaceutical Industry Associations (EFPIA). Arch Osteoporos 8, 136, doi:10.1007/s11657-013-0136-1 (2013).

9 Siris, E. S. et al. Bone mineral density thresholds for pharmacological intervention to prevent fractures. Arch Intern Med 164, 1108-1112, doi:10.1001/archinte.164.10.1108 (2004).

10 Body, J. J. Increased fracture rate in women with breast cancer: a review of the hidden risk. BMC Cancer 11, 384, doi:10.1186/1471-2407-11-384 (2011).

11 Andreasen, C. M. et al. Understanding Age-Induced Cortical Porosity in Women: The Accumulation and Coalescence of Eroded Cavities Upon Existing Intracortical Canals Is the Main Contributor. J Bone Miner Res 33, 606-620, doi:10.1002/jbmr.3354 (2018). Drake, M. T., Clarke, B. L. \& Lewiecki, E. M. The Pathophysiology and Treatment of Osteoporosis. Clin Ther 37, 1837-1850, doi:10.1016/j.clinthera.2015.06.006 (2015).

13 Bell, K. L. et al. Regional differences in cortical porosity in the fractured femoral neck. Bone 24, 57-64 (1999).

14 Zebaze, R. \& Seeman, E. Cortical bone: a challenging geography. J Bone Miner Res 30, 24-29, doi:10.1002/jbmr.2419 (2015).

15 Chen, H., Zhou, X., Shoumura, S., Emura, S. \& Bunai, Y. Age- and gender-dependent changes in three-dimensional microstructure of cortical and trabecular bone at the human femoral neck. Osteoporosis Int 21, 627-636, doi:10.1007/s00198-009-0993-z (2010).

16 lori, G. et al. Cortical thinning and accumulation of large cortical pores in the tibia reflect local structural deterioration of the femoral neck. Bone 137, 115446, doi:10.1016/j.bone.2020.115446 (2020).

17 Nishiyama, K. K., Macdonald, H. M., Buie, H. R., Hanley, D. A. \& Boyd, S. K. Postmenopausal women with osteopenia have higher cortical porosity and thinner cortices at the distal radius and tibia than women with normal aBMD: an in vivo HR-pQCT study. J Bone Miner Res 25, 882-890, doi:10.1359/jbmr.091020 (2010).

18 Iori, G. et al. BMD-based assessment of local porosity in human femoral cortical bone. Bone 114, 50-61, doi:10.1016/j.bone.2018.05.028 (2018). 
19 Schuit, S. C. et al. Fracture incidence and association with bone mineral density in elderly men and women: the Rotterdam Study. Bone 34, 195-202 (2004).

20 Wainwright, S. A. et al. Hip fracture in women without osteoporosis. J Clin Endocrinol Metab 90, 2787-2793, doi:10.1210/jc.2004-1568 (2005).

21 Hans, D. \& Baim, S. Quantitative Ultrasound (QUS) in the Management of Osteoporosis and Assessment of Fracture Risk. Journal of Clinical Densitometry 20, 322-333, doi:10.1016/j.jocd.2017.06.018 (2017).

22 Nicholson, P. F. Ultrasound and the biomechanical competence of bone. IEEE Trans Ultrason Ferroelectr Freq Control 55, 1539-1545, doi:10.1109/TUFFC.2008.830 (2008).

23 Grimal, Q. \& Laugier, P. Quantitative Ultrasound Assessment of Cortical Bone Properties Beyond Bone Mineral Density. Irbm 40, 16-24, doi:10.1016/j.irbm.2018.10.006 (2019).

24 Iori, G., Du, J., Hackenbeck, J., Kilappa, V. \& Raum, K. Estimation of Cortical Bone Microstructure From Ultrasound Backscatter. IEEE Trans Ultrason Ferroelectr Freq Control 68, 1081-1095, doi:10.1109/TUFFC.2020.3033050 (2021).

25 Lewiecki, E. M. Pulse-echo Ultrasound Identifies Caucasian and Hispanic Women at Risk for Osteoporosis. J Clin Densitom, doi:10.1016/j.jocd.2020.04.002 (2020).

26 Karjalainen, J. P., Riekkinen, O. \& Kroger, H. Pulse-echo ultrasound method for detection of post-menopausal women with osteoporotic BMD. Osteoporos Int 29, 1193-1199, doi:10.1007/s00198-018-4408-x (2018).

27 Behrens, M. et al. The Bindex ((R)) ultrasound device: reliability of cortical bone thickness measures and their relationship to regional bone mineral density. Physiol Meas 37, 15281540, doi:10.1088/0967-3334/37/9/1528 (2016).

28 Diez-Perez, A. et al. Radiofrequency echographic multi-spectrometry for the in-vivo assessment of bone strength: state of the art-outcomes of an expert consensus meeting organized by the European Society for Clinical and Economic Aspects of Osteoporosis, Osteoarthritis and Musculoskeletal Diseases (ESCEO). Aging Clin Exp Res 31, 1375-1389, doi:10.1007/s40520-019-01294-4 (2019).

29 Garg, M. K. \& Kharb, S. Dual energy X-ray absorptiometry: Pitfalls in measurement and interpretation of bone mineral density. Indian J Endocrinol Metab 17, 203-210, doi:10.4103/2230-8210.109659 (2013).

30 Whittier, D. E. et al. Guidelines for the assessment of bone density and microarchitecture in vivo using high-resolution peripheral quantitative computed tomography. Osteoporos Int 31, 1607-1627, doi:10.1007/s00198-020-05438-5 (2020).

31 Kroker, A. et al. Distal skeletal tibia assessed by HR-pQCT is highly correlated with femoral and lumbar vertebra failure loads. J Biomech 59, 43-49, doi:10.1016/j.jbiomech.2017.05.011 (2017).

32 Iori, G. et al. Large cortical bone pores in the tibia are associated with proximal femur strength. PLoS One 14, e0215405, doi:10.1371/journal.pone.0215405 (2019).

33 Whittier, D. E., Burt, L. A., Hanley, D. A. \& Boyd, S. K. Sex- and Site-Specific Reference Data for Bone Microarchitecture in Adults Measured Using Second-Generation HR-pQCT. J Bone Miner Res 35, 2151-2158, doi:10.1002/jbmr.4114 (2020).

34 Foldes, A. J., Rimon, A., Keinan, D. D. \& Popovtzer, M. M. Quantitative ultrasound of the tibia: a novel approach for assessment of bone status. Bone 17, 363-367, doi:10.1016/s87563282(95)00244-8 (1995).

35 Schneider, J. et al. In Vivo Measurements of Cortical Thickness and Porosity at the Proximal Third of the Tibia Using Guided Waves: Comparison with Site-Matched Peripheral Quantitative Computed Tomography and Distal High-Resolution Peripheral Quantitative Computed Tomography. Ultrasound Med Biol 45, 1234-1242, doi:10.1016/j.ultrasmedbio.2019.01.008 (2019).

36 Minonzio, J. G. et al. Ultrasound-Based Estimates of Cortical Bone Thickness and Porosity Are Associated With Nontraumatic Fractures in Postmenopausal Women: A Pilot Study. J Bone Miner Res 34, 1585-1596, doi:10.1002/jbmr.3733 (2019). 
37 Renaud, G., Kruizinga, P., Cassereau, D. \& Laugier, P. In vivo ultrasound imaging of the bone cortex. Phys Med Biol 63, 125010, doi:10.1088/1361-6560/aac784 (2018).

38 Roux, C. et al. Assessment of non-vertebral fracture risk in postmenopausal women. Ann Rheum Dis 66, 931-935, doi:10.1136/ard.2006.064071 (2007).

39 Bjornerem, A. et al. Fracture risk and height: an association partly accounted for by cortical porosity of relatively thinner cortices. J Bone Miner Res 28, 2017-2026, doi:10.1002/jbmr.1934 (2013).

40 Finigan, J. et al. Risk factors for vertebral and nonvertebral fracture over 10 years: a population-based study in women. J Bone Miner Res 23, 75-85, doi:10.1359/jbmr.070814 (2008).

41 Minonzio, J. G. et al. Ultrasound-based estimates of cortical bone thickness and porosity are associated with non-traumatic fractures in postmenopausal women: A pilot study. $J$ Bone Miner Res, doi:10.1002/jbmr.3733 (2019).

42 Moayyeri, A. et al. Quantitative ultrasound of the heel and fracture risk assessment: an updated meta-analysis. Osteoporos Int 23, 143-153, doi:10.1007/s00198-011-1817-5 (2012).

43 Stein, E. M. et al. Clinical assessment of the $1 / 3$ radius using a new desktop ultrasonic bone densitometer. Ultrasound Med Biol 39, 388-395, doi:10.1016/j.ultrasmedbio.2012.09.024 (2013).

44 Adami, G. et al. Radiofrequency echographic multi spectrometry for the prediction of incident fragility fractures: A 5-year follow-up study. Bone 134, 115297, doi:10.1016/j.bone.2020.115297 (2020).

45 Vallet, Q., Bochud, N., Chappard, C., Laugier, P. \& Minonzio, J. G. In Vivo Characterization of Cortical Bone Using Guided Waves Measured by Axial Transmission. IEEE Trans Ultrason Ferroelectr Freq Control 63, 1361-1371, doi:10.1109/TUFFC.2016.2587079 (2016).

46 Peralta, L. et al. Bulk Wave Velocities in Cortical Bone Reflect Porosity and Compression Strength. Ultrasound Med Biol 47, 799-808, doi:10.1016/j.ultrasmedbio.2020.11.012 (2021).

47 Granke, M., Makowski, A. J., Uppuganti, S. \& Nyman, J. S. Prevalent role of porosity and osteonal area over mineralization heterogeneity in the fracture toughness of human cortical bone. J Biomech 49, 2748-2755, doi:10.1016/j.jbiomech.2016.06.009 (2016).

48 Granke, M. et al. Change in porosity is the major determinant of the variation of cortical bone elasticity at the millimeter scale in aged women. Bone 49, 1020-1026, doi:10.1016/j.bone.2011.08.002 (2011).

49 Gluer, C. C. et al. Accurate assessment of precision errors: how to measure the reproducibility of bone densitometry techniques. Osteoporos Int 5, 262-270, doi:10.1007/BF01774016 (1995).

50 Burghardt, A. J., Buie, H. R., Laib, A., Majumdar, S. \& Boyd, S. K. Reproducibility of direct quantitative measures of cortical bone microarchitecture of the distal radius and tibia by HRpQCT. Bone 47, 519-528, doi:10.1016/j.bone.2010.05.034 (2010).

51 Li, H. D., Xu, Q. S. \& Liang, Y. Z. libPLS: An integrated library for partial least squares regression and linear discriminant analysis. Chemometr Intell Lab 176, 34-43, doi:10.1016/j.chemolab.2018.03.003 (2018).

\section{Acknowledgements}

This work was supported by the German Ministry of Science and Education (BMBF KMUi grant no. 13GW0234) and by the German Ministry of Economic Affairs and Energy (BMWi grant no. 03THW08H01). The HR-pQCT was funded by Deutsche Forschungsgemeinschaft (DFG, German Research Foundation) in the framework of the "Major Research Instrumentation" funding program as defined in Art. 91b of the Basic Law, application number: INST 335/555-1. We gratefully thank Gampt $\mathrm{GmbH}$ and exceeding solutions $\mathrm{GmbH}$ for their contributions to develop the CortBS data acquisition software and Mathis Manzel for support in the HR-pQCT data analysis. 


\section{Author contributions}

GA and KR planned the study. GA and HNM conducted the data acquisition. KR developed the CortBS data analysis. GA, JM, HNM, and KR performed the analysis, and interpretation of results; KR drafted the manuscript. GA, HNM, JM, and KR reviewed, edited, and approved the manuscript.

\section{Competing interests}

$\mathrm{JM}$ is employee of poroUS $\mathrm{GmbH}$, a startup developing the CortBS technology. KR is inventor on the patent applications (EP3641657A1, US 2020/0129140, CN110769754A and JP 2019-570514) describing the CortBS technology. 


\section{Supplementary Files}

This is a list of supplementary files associated with this preprint. Click to download.

- Supplementarymaterialsformanuscript.pdf 